\title{
Atrial Septal Defect with Pulmonary Stenosis: Staged or Simultaneous Intervention?
}

\author{
Setiawan Widodo*, Lucia Kris Dinarti, Budi Yuli Setianto \\ Department of Cardiology and Vascular Medicine, Faculty of Medicine, Universitas Gadjah Mada - \\ Dr. Sardjito Hospital, Yogyakarta, Indonesia. \\ *correspondence: stiawanwidodo@gmail.com
}

\begin{abstract}
Background: Congenital heart disease (CHD) is one of the most common inborn defects. The incidence is about $0.5-1.2 \%$ among newborns. Pulmonary valve stenosis and atrial septal defect are the common forms of congenital heart disease; however, their association is relatively rare. When the two conditions are present simultaneously, significant left-to-right shunt is often hampered by the outflow obstruction, which protects the pulmonary bed until adulthood.

Aims: This case reported the indication for atrial septal defect closure and pulmonary stenosis intervention. This paper also discussess whether simultaneous or staged intervention for both congenital heart malformations is recommended.

Case: Male, 20 years old, with the chief complain dispnea. Since childhood, patient suffered from dispnea, fatique, easily get sick without bluish discolorisation. At that time, patient had been diagnosed with congenital heart disease, but patient did not routinely visit doctor. Two years ago, patient complained worsening of dispnea, dispnea on effort, orthopnea and fatique. Patient did not complained peripheral oedema. Several examinations were performed to know severity of stenosis, hemodynamic condition, shunt and MAPCA. From the examination, patient had diagnosed with secundum atrial septal deffect (ASD), with severe valvular pulmonary stenosis (PS) with MAPCA from descendent aorta into main pulmonary artery. The patient was planned to have an ASD closure and PS resection. Finally, the surgery for ASD closure and pulmonary stenosis resection were performed simultaneously to this patient.

Discussion: The association of pulmonary valve stenosis and a significant ASD is relatively rare. When PS and ASD occur together, the interatrial shunt could be patent foramen ovale or ostium secundum ASD. Obstruction of the right ventricle (RV) outflow is usually caused by stenosis of the pulmonic valve (PV) and less commonly of the pulmorary artery (PA) and its branches. The pathophysiology of PS in association with ASD depends upon the degree of obstruction of RV outflow, the distensibility of the hypertrophied RV and the size of the interatrial communication. Patients can be classified into two subclasses: patients with severe PV stenosis and small RTLS via PFO; and patients with a large left to right shunt (LTRS) via a large ASD and mild to moderate obstruction to RV outflow. In patients with a large ASD and mild to moderate PS, the indication for intervention is when the ratio of pulmonary to systolic cardiac output is greater than 1.5 , or when there is evidence of RV volume overload, and heart failure. The decision to combine the pulmonary valve dilation and device closure of ASD depends upon various factors: size of ASD, adequate ASD rims, degree of pressure gradient across the pulmonary valve. Surgery is indicated if the pecutaneous intervention fails or is not feasible.

Conclusion: Patient had been performed surgery for ASD closure and pulmonary stenosis resection. There are no guidelines that describing clearly about staged or simultaneous intervention in atrial septal defect with pulmonary stenosis. There are some consideration whether to perform staged or symultaneous intervention.
\end{abstract}

Keywords: atria septal defect; pulmonary stenosis; trilogy of faloot 